\title{
XMM-Newton observations of the supernova remnant IC 443
}

\section{Evidence of stellar ejecta in the inner regions}

\author{
E. Troja ${ }^{1,2}$, F. Bocchino ${ }^{3}$, M. Miceli ${ }^{3}$, and F. Reale ${ }^{2,3}$ \\ 1 INAF - Istituto di Astrofisica Spaziale e Fisica Cosmica, Sezione di Palermo, via Ugo la Malfa 153, 90146 Palermo, Italy \\ e-mail: nora@ifc.inaf.it \\ 2 Dipartimento di Scienze Fisiche ed Astronomiche, Sezione di Astronomia, Università di Palermo, Piazza del Parlamento 1, \\ 90134 Palermo, Italy \\ 3 INAF - Osservatorio Astronomico di Palermo, Piazza del Parlamento 1, 90134 Palermo, Italy
}

Received 22 November 2007 / Accepted 29 March 2008

\section{ABSTRACT}

\begin{abstract}
Aims. We investigate the spatial distribution of the physical and chemical properties of the hot X-ray emitting plasma of the supernova remnant IC 443, to derive important constraints on its ionization stage, on the progenitor supernova explosion, on the age of the remnant, and its physical association with a close pulsar wind nebula.

Methods. We present XMM-Newton images of IC 443, a median photon energy map, silicon and sulfur equivalent width maps, and a spatially resolved spectral analysis of a set of homogeneous regions.

Results. The hard X-ray thermal emission (1.4-5.0 keV) of IC 443 displays a centrally-peaked morphology, its brightness peaks being associated with hot $(k T>1 \mathrm{keV}) \mathrm{X}$-ray emitting plasma. A ring-shaped structure, characterized by high values of equivalent widths and median photon energy, encloses the PWN. Its hard X-ray emission is spectrally characterized by a collisional ionization equilibrium model, and strong emission lines of $\mathrm{Mg}, \mathrm{Si}$, and $\mathrm{S}$, requiring oversolar metal abundances. Dynamically, the location of the ejecta ring suggests an SNR age of $\sim 4000 \mathrm{yr}$. The presence of overionized plasma in the inner regions of IC 443 , addressed in previous works, is much less evident in our observations.
\end{abstract}

Key words. ISM: supernova remnants - ISM: individual objects: IC 443 - pulsars: individual: CXOU J061705.3+222127 X-rays: ISM

\section{Introduction}

The Galactic SNR IC 443 (G189.1+3.0) has an optical and radio double shell morphology (Leahy 2004; Reich et al. 2003; Braun \& Strom 1986; Duin \& van der Laan 1975). Signatures of interaction with a dense and complex environment are visible at radio (e.g. Snell et al. 2005; Denoyer 1977) and IR wavelengths (Neufeld \& Yuan 2008; Rho et al. 2001, and references therein). In the northeast the shock front has been decelerated by the encounter with a H I cloud (Rho et al. 2001; Dickel et al. 1989; Denoyer 1978). A giant molecular cloud, mapped by Cornett et al. (1977), is located in the foreground, and is interacting with the remnant at several positions along the southern rim (as schematically represented in Fig. 9 of Troja et al. 2006). IC 443 is also a source of $\gamma$-rays (Esposito et al. 1996; Sturner \& Dermer 1995). Interestingly, in the same region of sky where $\mathrm{OH}(1720 \mathrm{MHz})$ maser emission is detected (Hewitt et al. 2006; Hoffman et al. 2003; Claussen et al. 1997), Albert et al. (2007) discovered a source of very high energy $\gamma$-ray emission, possibly originating in the interactions between cosmic rays accelerated in IC 443 and the dense molecular cloud.

Very interesting features and a peculiar morphology are displayed in the X-ray band (Bykov et al. 2008, 2005; Bocchino \& Bykov 2003; Asaoka \& Aschenbach 1994; Wang et al. 1992; Petre et al. 1988). The bulk of its X-ray emission is thermal, fairly well described with a two components ionization equilibrium model of temperatures $\sim 0.2-0.3 \mathrm{keV}$ (hereafter the "cold component") and $\gtrsim 1.0 \mathrm{keV}$ (hereafter the "hot component";
Troja et al. 2006; Asaoka \& Aschenbach 1994; Petre et al. 1988). A source of very hard X-ray emission, identified as a pulsar wind nebula (PWN), lies close to the SNR's southern rim and is possibly associated with the remnant (Gaensler et al. 2006; Bocchino \& Bykov 2001; Olbert et al. 2001).

On the basis of its X-ray properties, as observed by ROSAT, IC 443 has been classified as a mixed morphology (MM) SNR. MM SNR main features were described by Rho \& Petre (1998) as follows: 1) the X-ray emission is thermal and centrally peaked, with little or no evidence of a limb-brightened X-ray shell; 2) it arises primarily from swept-up interstellar material, not from ejecta; 3 ) a flat temperature radial profile, and a constant or increasing density toward the remnant center; 4) a physical association with dense molecular clouds.

XMM-Newton high resolution observations of IC 443, presented by Troja et al. (2006), resolved a limb-brightened shell structure in the very soft X-ray band $(0.3-0.5 \mathrm{keV})$, and reported first evidence of $\mathrm{Mg}, \mathrm{Si}$ and $\mathrm{S}$ enhanced abundances. In addition, XMM-Newton and Chandra observations of several other MM SNRs unveiled very steep X-ray brightness profiles and multi-component X-ray-emitting plasma with enhanced abundances (Lazendic \& Slane 2006; Shelton et al. 2004). More recent results suggest a non negligible presence of metal enrichment gradients and stellar ejecta inside at least $\sim 50 \%$ of all known MM SNRs.

Strong Si and S lines in the IC 443 X-ray spectrum were first reported by Petre et al. (1988), and then studied in detail by Kawasaki et al. (2002). In the latter work the X-ray emission 
from IC 443 was described by a two temperature model, with a $0.2 \mathrm{keV}$ component in ionization non-equilibrium (NEI) and a $1.0 \mathrm{keV}$ component in collisional ionization equilibrium (CIE). Narrow Gaussians were used to reproduce the most prominent lines. The element abundances were all below the solar value, as expected for X-ray emission from the shocked ISM. From the relative strengths of Si and S lines, Kawasaki et al. (2002) derived an ionization temperature of $\sim 1.5 \mathrm{keV}$, significantly above the $1.0 \mathrm{keV}$ continuum temperature. Those results led to the hypothesis of an overionized thermal plasma.

Using XMM-Newton observations of the SNR IC 443, we presented a detailed analysis of the soft X-ray thermal emission, mainly originating from the shocked ISM (see Troja et al. 2006). Unlike Kawasaki et al. (2002) our spectral results gave a soft $\mathrm{X}$-ray component $(k T \sim 0.2-0.3 \mathrm{keV})$ near the equilibrium condition $\left(\tau \sim 10^{12} \mathrm{~cm}^{-3} \mathrm{~s}\right)$ in most of the analyzed regions. The hot component $(k T \gtrsim 1.0 \mathrm{keV})$ was in full equilibrium and showed a high metallicity.

The present work focuses on the properties of the hot X-ray thermal component, and is aimed at 1) addressing the high metal abundance plasma inside the remnant and its ionization stage; 2) checking whether the abundance pattern of the hot X-ray emitting plasma is consistent with a core-collapse $\mathrm{SN}$ as a progenitor, as already hinted at by the presence of the PWN.

The paper is organized as follows: observations are described in Sect. 2; results from imaging and spectral analysis are presented in Sect. 3. In Sect. 4 we discuss their implications for the remnant ionization stage (Sect. 4.1), the SN progenitor (Sect. 4.2), and the age of IC 443 (Sect. 4.3). A summary of our conclusions is presented in Sect. 5. Throughout the paper, the quoted uncertainties are at the $90 \%$ confidence level (Lampton et al. 1976), unless otherwise stated.

\section{Observations and data reduction}

We analyzed four pointings XMM-Newton observations of IC 443, performed during the $\mathrm{Cal} / \mathrm{PV}$ phase on 2000 September 25-28 and previously described in Troja et al. (2006) and Bocchino \& Bykov (2003). We added a more recent and very deep XMM-Newton observation, taken on 2006 March 30 (ObsID 0301960101, P.I. F. Bocchino), and centered on the southeastern side of the remnant at $\mathrm{RA}(\mathrm{J} 2000)=$ $06^{\mathrm{h}} 18^{\mathrm{m}} 04^{\mathrm{s}}, \operatorname{Dec}(\mathrm{J} 2000)=+22^{\circ} 27^{\prime} 33^{\prime \prime}$ (see also Bykov et al. 2008).

Our work is focused on the analysis of EPIC MOS data. We used the Science Analysis System software (SAS,version 7.0) for data processing and XSPEC v.11.3 (Arnaud 1996) for spectral analysis.

Data were screened to remove spurious events and time intervals with heavy proton flaring. After the particle background removal, the total exposures for the MOS cameras (MOS1+MOS2) were $\sim 170 \mathrm{ks}$ and $\sim 140 \mathrm{ks}$ for the 2000 and 2006 data sets, respectively.

In order to estimate the Galactic background we used a set of blank sky observations performed as part of the Galactic Plane Survey (GPS, P.I. Parmar). We verified that, in the energy band $(0.3-5.0 \mathrm{keV})$ selected for the analysis, our results are not sensitive to the background subtraction (either GPS or high Galactic latitude blank fields). We refer to our previous paper (Troja et al. 2006) for more details about the observations and the data reduction techniques we used.

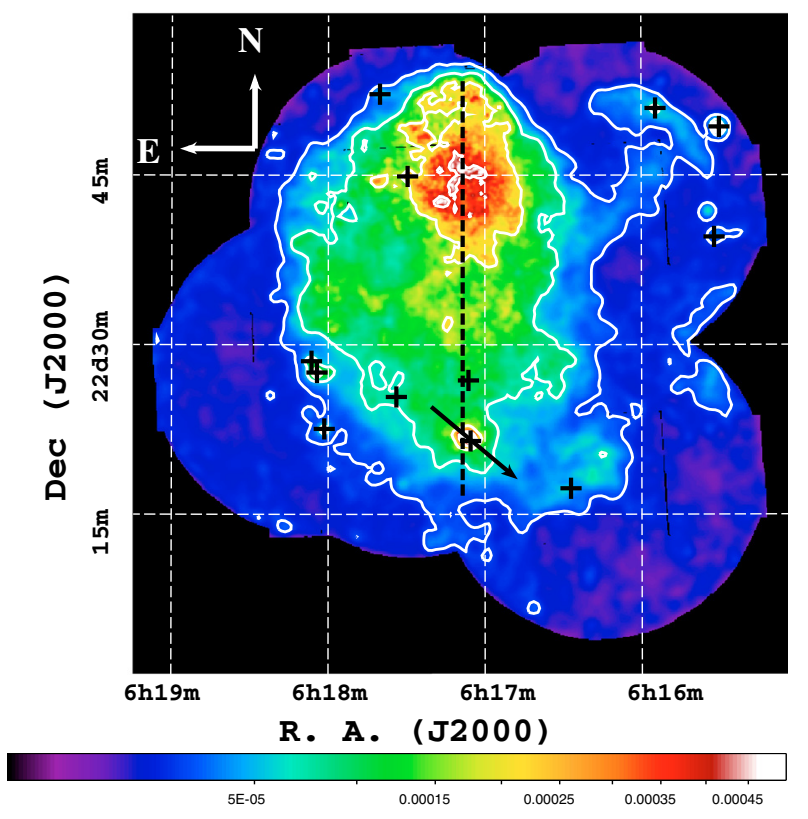

Fig. 1. X-ray count rate image of IC 443 in the $1.4-5.0 \mathrm{keV}$ energy band. Units are cts s${ }^{-1} \mathrm{bin}^{-1}$. The image was adaptively smoothed using $\sigma_{\min }=5^{\prime \prime}$ and $\sigma_{\max }=20^{\prime \prime}$; the bin size is $5^{\prime \prime}$. Contour levels correspond to $0.4,1.0,2.7$, and $5.8 \times 10^{-2} \mathrm{cts} \mathrm{s}^{-1} \mathrm{arcmin}^{-2}$. Black crosses mark the position of the 12 hard X-ray point sources identified by Bocchino \& Bykov (2003). The dashed line traces the symmetry axis of the X-ray emission in the hard band; the black arrow indicates the putative direction of motion of the PWN, according to Gaensler et al. (2006).

\section{Results}

\subsection{X-ray Images}

Figure 1 shows the composite image of IC 443 in the hard 1.4-5.0 keV energy band, where the contribution of the cold $\mathrm{X}$-ray emitting plasma is negligible (see Troja et al. 2006). The image is background subtracted, exposure and vignetting corrected. We reported the position of the 12 hard X-ray point sources detected by Bocchino \& Bykov (2003).

The hard band morphology does not resemble IC 443 emission in other bands (optical, radio, IR and soft X-ray) and, in the plane of the image, it appears inwardly concetrated with respect to the cold X-ray emitting plasma.

The hard X-ray thermal emission is dominated by an elongated axial symmetric structure, having its surface brightness peak in the northeastern (NE) quadrant. The symmetry axis, traced by the dashed line in Fig. 1, does not match with the plerion wind nebula $(\mathrm{PWN})$ direction of motion $\left(-130^{\circ} \mathrm{N}\right.$ through E, Gaensler et al. 2006), as indicated by the arrow in Fig. 1.

The X-ray surface brightness is higher $(\Sigma \geq$ $0.012 \mathrm{cts} \mathrm{s}^{-1} \operatorname{arcmin}^{-2}$ ) in the subshell A (following the naming convention of Braun \& Strom 1986; see also Fig. 5 of Troja et al. 2006), which is associated with the presence of the neutral cloud in the NE, while it appears strongly reduced $(\Sigma<$ $0.012 \mathrm{cts} \mathrm{s}^{-1} \operatorname{arcmin}^{-2}$ ) in the western part (subshell B), where the blast wave is expanding in a more rarefied $\left(n_{0} \sim 0.25 \mathrm{~cm}^{-3}\right)$ and homogeneous medium.

\subsection{Median energy map}

The large XMM-Newton sensitivity and collecting area enabled us to create a high resolution image of the median photon energy $E_{50}$ in the $1.4-5.0 \mathrm{keV}$ band. In the selected energy range 


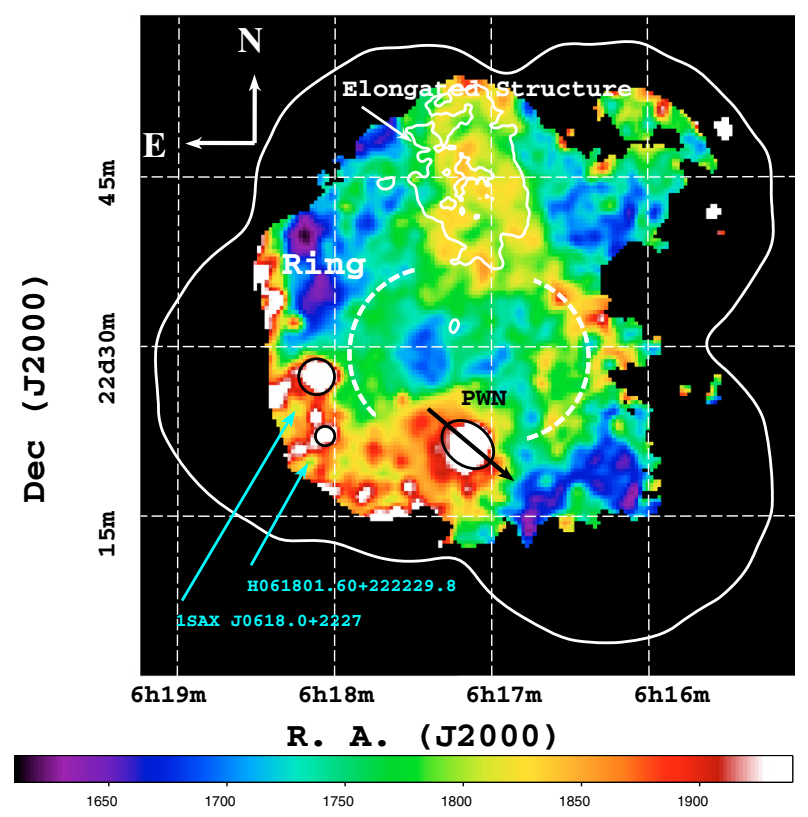

Fig. 2. Median photon energy map in the hard band (1.4-5.0 keV). Units are $\mathrm{eV}$. Hard X-ray emission contours, corresponding to 2.7 and $5.8 \times 10^{-2} \mathrm{cts} \mathrm{s}^{-1} \mathrm{arcmin}^{-2}$, are overlaid. Noisy $(S / N<3)$ regions inside the FOV, delimited by a white solid line, were masked. The bin size is $20^{\prime \prime}$ and the smoothing width is $\sigma=20^{\prime \prime}$. The two dashed semi-circles guide the eye to notice a ring-shaped structure, which is more evident in the $E W$ maps shown in Fig. 3. The location of some sources, partially responsible for the high median energy in the SE region, is indicated with green arrows.

the median energy is mostly influenced by the parameters of the hot X-ray component, being unaffected by spectral variations of the colder one. For a plasma in ionization equilibrium and with solar abundances (model MEKAL in XSPEC) we verified that the $E_{50}$ distribution represents a powerful tracer of temperature variations, especially for $k T \geq 1 \mathrm{keV}$.

Figure 2 shows the median energy map of the X-ray emitting plasma in the 1.4-5.0 keV band. For comparison, we overlaid the brightest hard X-ray emission contours of Fig. 1. The white dashed line guides the eye to identify a ring-shaped structure, which will be discussed in Sect. 3.3.

The median photon energy image clearly shows a north-tosouth elongated structure, spatially corresponding to the brightest emission features in the hard energy band (contours in Fig. 2). The region of high median energy $\left(E_{50} \gtrsim 1.9 \mathrm{keV}\right)$ along the southeast edge can be partially attributed to the emission of the PWN and of the other point sources (Bocchino \& Bykov 2003), but it appears to have a much larger extent, suggestive of an extended hard emission. The nature of the hard X-ray source 1SAX J0618.0+2227 (also marked in Fig. 2) has been extensively investigated (Bykov et al. 2008, 2005; Bocchino \& Bykov 2000) and interpreted in the framework of interaction of a molecular cloud with a fast moving SN ejecta fragment. The study of the diffuse hard emission revealed in Fig. 2 is beyond the scope of this work, however we note that it may have a similar physical origin, probably arising from the interaction of the SNR with the structured cloud (van Dishoeck et al. 1993; Burton et al. 1990).

\subsection{Equivalent width images}

IC $443 \mathrm{X}$-ray emission is primarily thermal and its spectrum is dominated by prominent atomic emission lines. Its global
Table 1. Energy bands selected for the equivalent width images.

\begin{tabular}{lccc}
\hline \hline Elements & $\begin{array}{c}\text { Lines } \\
(\mathrm{eV})\end{array}$ & $\begin{array}{c}\text { Low continuum } \\
(\mathrm{eV})\end{array}$ & $\begin{array}{c}\text { High continuum } \\
(\mathrm{eV})\end{array}$ \\
\hline $\mathrm{Si} \ldots \ldots$ & $1790-2060$ & $1630-1690$ & $2180-2320$ \\
$\mathrm{~S} \ldots \ldots$. & $2360-2680$ & $2180-2320$ & $3350-3520$ \\
\hline
\end{tabular}

spectrum can be described with a phenomenological model, consisting of two bremsstrahlung components for the continuum ( $k T \sim 0.2 \mathrm{keV}$ and $k T \sim 1.1 \mathrm{keV}$ ) plus Gaussian features reproducing the line emission.

We explored the spatial distribution of the hard X-ray line emission by constructing equivalent width $(E W)$ images, often used as powerful tracers of stellar ejecta fragments in SNRs (Hwang et al. 2000; Gotthelf et al. 2001; Park et al. 2003a; Cassam-Chenaï et al. 2004; Miceli et al. 2006).

We selected an energy band for each emission line, combining the $\operatorname{He} \alpha$ and Ly $\alpha$ emission of each element (e.g. Si XIII and $\mathrm{SiXIV)}$ in a single image to improve the statistics. We chose the energy band that optimizes the signal to noise $(S / N)$ ratio according to our best-fit phenomenological model.

$\mathrm{X}$-ray emission in the line energy band needs to be corrected for the underlying continuum emission. The correction is made pixel by pixel because the continuum may be spatially distributed in a different way from the line emission. In order to account for the continuum contamination, we selected two continuum bands located on either side of the line energy band. Their width was chosen to optimize the $S / N$ ratio, according to the recipe of Cassam-Chenaï et al. (2004).

This method was successfully applied to silicon and sulfur lines. We excluded the $\mathrm{H}$-like $\mathrm{Mg}$ line at $1.47 \mathrm{keV}$ since the underlying continuum emission cannot be correctly estimated. In this particular case, the low energy continuum band $(\sim 1.1 \mathrm{keV})$ is dominated by the soft spectral component and is still affected by the strong absorbing column gradients, due to the foreground molecular cloud.

Line and continuum band passes, selected for each spectral line of interest, are listed in Table 1.

Once an image in each energy band was generated, we estimated the continuum emission in the line band (see e.g. Cassam-Chenaï et al. 2004). In each pixel we computed the underlying continuum as a linear combination of the source emission in adjacent continuum bands:

$I_{\mathrm{c}}=\lambda \alpha_{\mathrm{c}}^{-} I_{\mathrm{c}}^{-}+(1-\lambda) \alpha_{\mathrm{c}}^{+} I_{\mathrm{c}}^{+}$

where $I_{\mathrm{c}}$ is the resulting continuum image, and $I_{\mathrm{c}}^{ \pm}$are the background subtracted images in the high and low continuum bands, respectively. The normalization coefficients, $\alpha_{\mathrm{c}}^{ \pm}$, were determined by the ratio of the model predicted counts and the source measured counts in the continuum bands, as in Cassam-Chenaï et al. (2004). The weighting factor $\lambda$ was tuned to minimize statistical fluctuations.

Narrow band images were created with a bin size of $30^{\prime \prime}$ to provide at least 10 counts, even in the fainter SNR regions.

In order to generate $E W$ images, the image in the line band was background and continuum subtracted and then divided by the estimated continuum image $I_{\mathrm{c}}$. Since this operation amplified statistical fluctuations, we had previously applied an adaptive smoothing with $30^{\prime \prime} \leq \sigma \leq 120^{\prime \prime}$ and a signal to noise ratio equal to 5 . We set the $E W$ to zero in those pixels with a very low estimated continuum $(<5 \%$ than the maximum value, see e.g. Park et al. 2003b). 

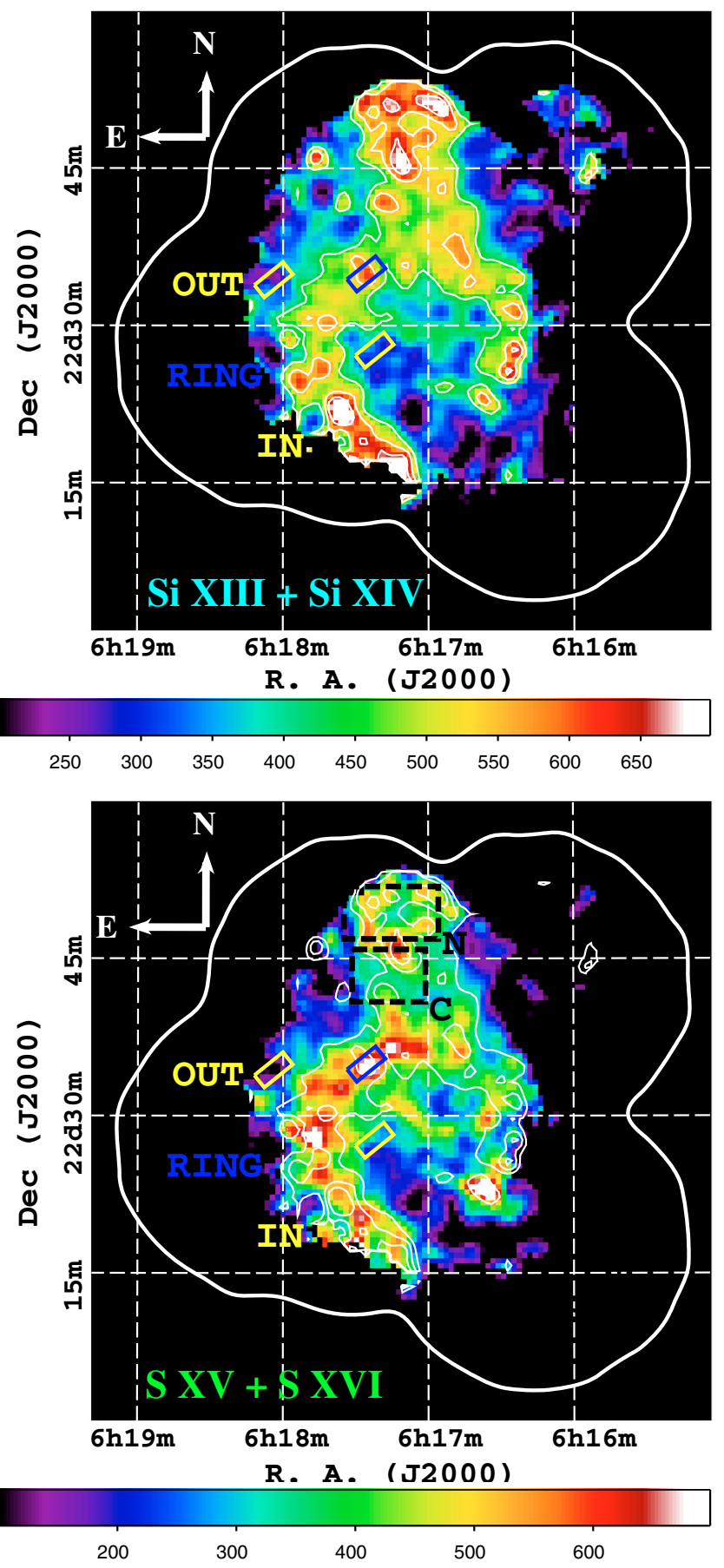

Fig. 3. Equivalent width images of Si (top panel) and S lines (bottom panel). Units are $\mathrm{eV}$. Noisy regions were masked, as described in the text. The thin white line mark the FOV. Contour levels corresponds to $450,550,650 \mathrm{eV}$ in the $\mathrm{Si} E W$ image. We also overlaid the three regions (named IN, RING, and OUT) selected for spectral analysis. The dashed boxes overlaid on the SEW map show the "North" and "Center" regions, the same as Kawasaki et al. (2002), and discussed on Sect. 3.4.1.

$\mathrm{Si}$ and $\mathrm{S} E W$ images are presented in Fig. 3, revealing an intriguing circular feature, $10^{\prime}$ in radius, in the southern part of the SNR. For a morphological comparison we superimposed Si $E W$ contours on the images, corresponding to 450,550 and $650 \mathrm{eV}$. The two images look very similar. The contours of high Si $E W$ match well with the bright $S E W$ features. In particular, we did not find any strong evidence of elemental stratification. Both the $E W$ maps show similar features to the median energy map: an elongated bright structure in the NE quadrant

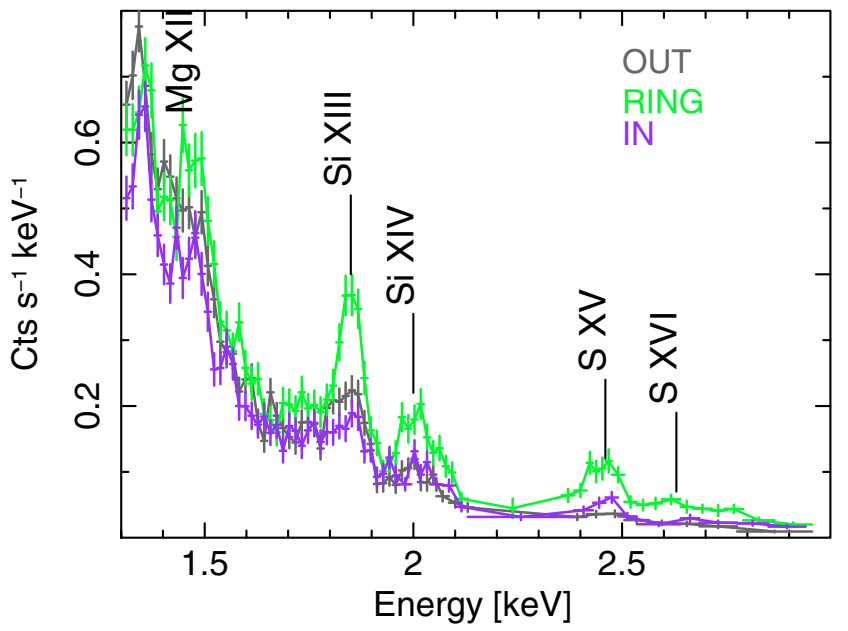

Fig. 4. A comparison of the three X-ray spectra at high energies, clearly showing brightest lines in the "ring" region respect with the external ("out") and the internal ("in") regions.

and a circular ring around the PWN region. A rather similar arclike feature has been observed in the Si EW map of the SNR N49 (Park et al. 2003a) and interpreted as the boundary of hot plasma, re-heated by the reverse shock. The Si arc in N49 is not around the central compact object but, as in the case of IC 443, it is located near the site of interaction with a molecular cloud complex, strengthening the hypothesis of an intense reverse shock, propagating back into the remnant.

The great advantage of $E W$ images is that they are not significantly affected by emission measure variations. However they do also depend on the plasma temperature and ionization age. In order to disentangle multiple effects, we proceeded with direct spatially resolved spectral analysis, using the median photon energy and the $E W$ images as guiding criteria for region selection.

\subsection{Spectral analysis}

Our aim was to confirm the bright X-ray features visible in Figs. 2 and 3, and give a quantitative characterization of their spectra. We extracted three EPIC MOS spectra, selected as representative on the basis of the median energy map and the $E W$ images. We selected homogeneous regions, marked in Fig. 3, characterized by small fluctuations $(\ll 5 \%)$ in the median photon energy and in the line $E W \mathrm{~s}$. Two of them, named "in" and "out", have low median energies, low EWs values, and lie inside and outside the ring structure. The third region, named "ring" and belonging to the ring structure, is particularly bright both in the median energy and in the $E W$ images.

Source and background event lists were corrected for vignetting effects with the task evigweight of the SAS package, and then spectra were extracted. We used the relevant on-axis ancillary response file and response matrices generated with the SAS task rmfgen. Spectra were grouped with a minimum of 25 counts per bin and the $\chi^{2}$ statistics was used. Figure 4 shows the three spectra at high energies, where striking differences in the properties of the hot X-ray emitting plasma are clearly visible. Prominent $\mathrm{Mg}, \mathrm{Si}$, and $\mathrm{S}$ lines characterize the "ring" spectrum, whilst line emission is much fainter in the other two regions. Figure 4 confirms that the identified features correspond to different physical conditions of the emitting plasma.

We modeled the spectra over the $0.5-5.0 \mathrm{keV}$ energy band with a NEI plus a CIE thermal component 


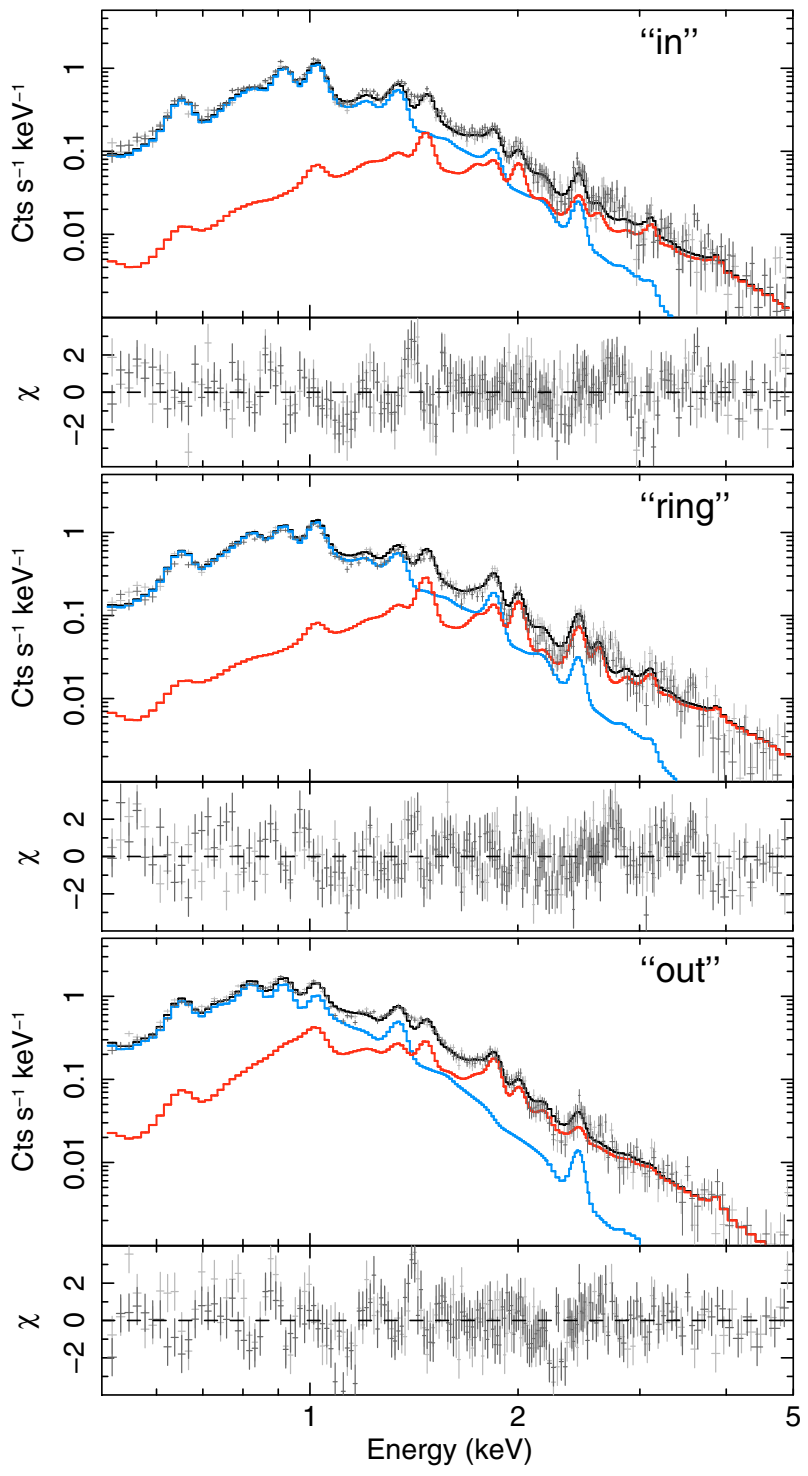

Fig. 5. EPIC MOS spectra of IC 443, selected as representative of the hot X-ray plasma properties, and their best-fit models overlaid. Panels a)-c) correspond to regions "in", "ring", and "out" in Fig. 3, respectively. The lower panel in each plot shows the residuals from the best-fit model.

(VPSHOCK+VMEKAL in XSPEC), as in Troja et al. (2006). MOS1 and MOS2 spectra were simultaneously fitted, leaving the normalization between the two instruments as a free parameter. We included a 5\% systematic error term accounting for the uncertainties in the calibration ${ }^{1}$. The absorbing column $N_{\mathrm{H}}$ was estimated by using the median photon energy in the soft band (cf. Troja et al. 2006, Fig. 6), and held fixed at the derived value.

Spectra and best-fit models are shown in Fig. 5. Table 2 reports the spectral properties (median photon energy $E_{50}, \mathrm{Si}$ and $\mathrm{S} E W \mathrm{~s}$ ), and the best-fit spectral parameters for each region.

\subsubsection{Overionization}

Taking advantage of the large XMM-Newton effective area at high energies, we checked the overionization claim made by Kawasaki et al. (2002, 2005). For this purpose, we extracted two

1 The current EPIC MOS status of calibration is reported at http:// xmm.vilspa.esa.es/docs/documents/CAL-TN-0018.pdf
Table 2. Spectral parameters of the three analysed regions.

\begin{tabular}{|c|c|c|c|}
\hline Parameters & $\overline{\mathrm{IN}}$ & RING & $\overline{\text { OUT }}$ \\
\hline$E_{50}(\mathrm{keV})$ & 1.70 & 1.78 & 1.65 \\
\hline Si $E W(\mathrm{eV})$ & 310 & 550 & 290 \\
\hline $\mathrm{S} E W(\mathrm{eV})$ & 280 & 690 & 130 \\
\hline \multicolumn{4}{|c|}{ Cold component } \\
\hline$k T_{\mathrm{s}}(\mathrm{keV})$ & $0.44_{-0.03}^{+0.04}$ & $0.43 \pm 0.02$ & $0.32_{-0.02}^{+0.03}$ \\
\hline$\tau\left(10^{12} \mathrm{~cm}^{-3} \mathrm{~s}\right)$ & $1.7_{-0.06}^{+0.04}$ & $1.8 \pm 0.05$ & $2.5_{-1.0}^{+0.5}$ \\
\hline $\mathrm{O} / \mathrm{O}_{\odot} \ldots \ldots$ & $0.78_{-0.2}^{+0.16}$ & $0.68_{-0.08}^{+0.2}$ & $0.30 \pm 0.10$ \\
\hline $\mathrm{Ne} / \mathrm{Ne}_{\odot} \ldots$ & $0.91_{-0.10}^{+0.3}$ & $0.81_{-0.3}^{+0.12}$ & $0.35_{-0.05}^{+0.10}$ \\
\hline $\mathrm{Mg} / \mathrm{Mg}_{\odot} \ldots$ & $0.45_{-0.05}^{+0.09}$ & $0.35 \pm 0.05$ & $0.35 \pm 0.05$ \\
\hline $\mathrm{Si} / \mathrm{Si}_{\odot} \ldots \ldots$ & $0.26_{-0.11}^{+0.06}$ & $0.50_{-0.15}^{+0.10}$ & $<0.15$ \\
\hline $\mathrm{Fe} / \mathrm{Fe}_{\odot} \ldots \ldots \ldots$ & 0.10 & $0.20 \pm 0.03$ & $0.22 \pm 0.05$ \\
\hline $\operatorname{EM}_{\mathrm{s}}\left(10^{56} \mathrm{~cm}^{-3}\right) \ldots$ & 4.1 & 5.7 & 8.7 \\
\hline \multicolumn{4}{|c|}{ Hot component } \\
\hline$k T_{\mathrm{h}}(\mathrm{keV}) \ldots$ & $1.35_{-0.19}^{+0.4}$ & $1.45_{-0.15}^{+0.08}$ & $0.86 \pm 0.05$ \\
\hline $\mathrm{Mg} / \mathrm{Mg}_{\odot} \ldots \ldots$ & $2.6_{-1.1}^{+3}$ & $5.0_{-0.9}^{+3}$ & [1] \\
\hline $\mathrm{Si} / \mathrm{Si}_{\odot} \ldots$ & $1.1 \pm 0.3$ & $2.4 \pm 0.7$ & $0.67_{-0.11}^{+0.19}$ \\
\hline $\mathrm{S} / \mathrm{S}_{\odot} \ldots$ & $0.7 \pm 0.5$ & $2.3_{-0.7}^{+0.6}$ & $0.22_{-0.11}^{+0.12}$ \\
\hline $\mathrm{Fe} / \mathrm{Fe}_{\odot} \ldots$ & $<0.13$ & $<0.03$ & $<0.23$ \\
\hline $\operatorname{EM}_{\mathrm{h}}\left(10^{56} \mathrm{~cm}^{-3}\right) \ldots \ldots$ & 1.1 & 0.4 & 0.4 \\
\hline$\chi^{2} /$ d.o.f. . . . . . . . . . & $420 / 324$ & $497 / 343$ & $412 / 302$ \\
\hline
\end{tabular}

spectra from the "North" and "Center" regions (see Fig. 2 of Kawasaki et al. 2002), and performed the same comparison between the ionization and the electron continuum temperatures.

We selected the 2.2-5.0 keV energy range and we modeled the spectrum with a thermal component (model VMEKAL in XSPEC), fixing the $S$ abundance to zero. Three narrow Gaussians (centered at 2.46, 2.62 and $2.88 \mathrm{keV}$ ) were used to model the sulfur line emission. The intensity line ratio of the H-like and He-like ions (S XVI/S XV) is a function of the S ionization temperature, derived using the APED line emissivity database (Smith et al. 2001).

The absorption column densities were held fixed to the values of $0.60 \times 10^{22} \mathrm{~cm}^{-2}$ for the "North" spectrum and $0.68 \times$ $10^{22} \mathrm{~cm}^{-2}$ for the "Center" spectrum, as derived from the $N_{\mathrm{H}} / E_{50}$ calibration plot (Fig. 10 in Troja et al. 2006). We also tested the absorption column value of $0.74 \times 10^{22} \mathrm{~cm}^{-2}$ used by Kawasaki et al. (2002), but it does not affect the final result, as expected at these high energies.

Figure 6 shows the $68 \%, 90 \%$, and $99 \% \chi^{2}$ contours in the parameter space $\mathrm{SXVI} / \mathrm{SXV}-k T_{\mathrm{e}}$. The CIE is represented by the dashed line, tracing the condition $k T_{\mathrm{e}}=k T_{\mathrm{z}}$. In the "North" region we found evidence of an underionized plasma, in stark contrast with the findings of Kawasaki et al. (2002); in the "Center" region the derived ionization temperature is slightly higher than the the electron temperature, though consistent with it at the $90 \%$ confidence level. Therefore, a detailed analysis of the ionization state does not directly support the presence of an overionized plasma in the "North" region, and shows only a marginal $(1 \sigma)$ evidence of overionization in the innermost "Center" region. 


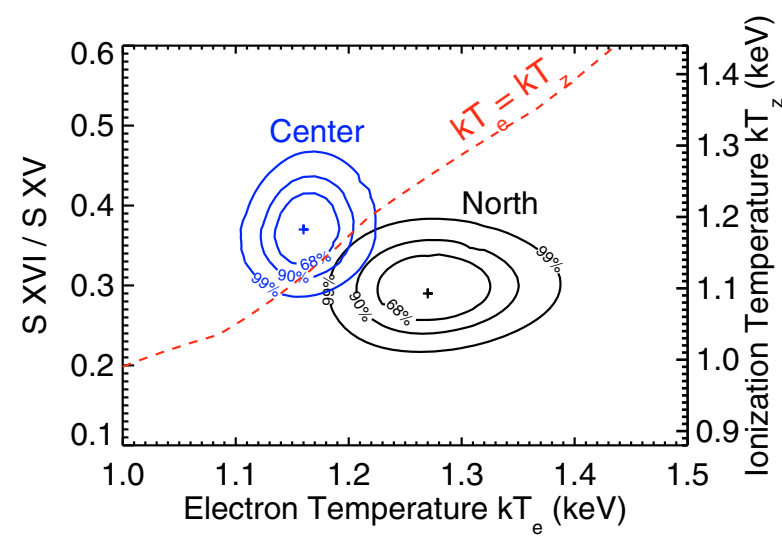

Fig. 6. Confidence level $\chi^{2}$ contours $(68 \%, 90 \%, 99 \%)$ of the line ratio $(\mathrm{S} X V \mathrm{I} / \mathrm{SXV})$ and electron temperature $\left(k T_{\mathrm{e}}\right)$ for the "North" and the "Center" region. The ionization temperature $k T_{z}$ is reported on the right $Y$-axis. The dashed line indicates the CIE state.

\section{Discussion}

\subsection{Presence of hot metal-rich plasma in IC 443}

Spatially-resolved spectral analysis confirms that the structures, revealed by the median photon energy and the $E W$ maps (see Sects. 3.2 and 3.3, respectively), are associated with a high temperature, high metallicity X-ray emitting plasma.

Our results, listed in Table 2, show that the cold plasma is characterized by a temperature of $0.3-0.4 \mathrm{keV}$, a high ionization timescale $\left(>10^{12} \mathrm{~cm}^{-3} \mathrm{~s}\right)$, and solar or undersolar abundances, as expected if the emission arises from the shocked ISM. The hot plasma is fully equilibrated. It has lower metal abundances in the "in" and "out" regions, in good agreement with previous findings for the hot component (Kawasaki et al. 2002; Petre et al. 1988). The temperature $T_{\mathrm{h}}$ and the abundances of $\mathrm{Mg}, \mathrm{Si}$, and $\mathrm{S}$ in the "ring" region are much higher, more similar to regions 2 , 6, and 9 analyzed in Troja et al. (2006). As shown in Figs. 2 and 3 , in the NE the hottest plasma appears concentrated in the innermost regions, whilst in the south it display a ring-shaped spatial distribution. Our spectral results agree with this picture, detecting a high temperature and a substantial metal-enrichment in correspondence to the "ring" region. The measured overabundant $\mathrm{Mg}$, Si and $\mathrm{S}$ are suggestive of SN ejecta emission.

IC 443 is a middle-aged remnant, thus the presence of ejecta at its evolutive stage is not expected. Models predict that most of the ejecta thermalized with the surrounding medium, and is no longer visible. However, XMM-Newton and Chandra high-resolution observations show a growing number of middleaged remnants $\left(\sim 10^{4} \mathrm{yr}\right)$ where metal rich ejecta are still detectable and contribute significantly to the X-ray emission, as in DEML71 (Hughes et al. 2003), N49B (Park et al. 2003a), and 0103-72.6 (Park et al. 2003b). Moreover, there are several cases among MM SNRs in which a soft component, associated with shocked ISM emission, and a hot metal enriched component have been resolved. For instance, Mavromatakis et al. (2004) described W63 thermal emission with a two temperatures plasma ( $k T_{1} \sim 0.2 \mathrm{keV}$ and $k T_{2} \sim 0.6 \mathrm{keV}$ ), showing overabundant $\mathrm{Mg}$, $\mathrm{Si}$ and $\mathrm{Fe}$ in a bright bar-like region near the geometric center of the SNR. Lazendic \& Slane (2006) resolved a two temperature thermal plasma in the inner regions of CTB 1 , a prototypical MM SNR, and they showed that the hard component $(k T \sim$ $0.8 \mathrm{keV}$ ) requires an oversolar $\mathrm{Mg}$ abundance.

Kawasaki et al. $(2002,2005)$ addressed the issue of overionization in the hot plasma of MM SNRs, finding that in 2 objects
Table 3. Relative abundances in the ring region: $[\mathrm{X} / \mathrm{Si}] /[\mathrm{X} / \mathrm{Si}]_{\odot}$.

\begin{tabular}{lcccccc}
\hline \hline Ratio & IC 443 & $20 M_{\odot}{ }^{a}$ & $25{M_{\odot}}^{a}$ & $30 M_{\odot}{ }^{a}$ & $\mathrm{PDDe}^{b}$ & $\mathrm{DDTe}^{b}$ \\
\hline $\mathrm{Mg} / \mathrm{Si} \ldots$ & $2.1_{-0.7}^{+1.4}$ & 0.16 & 0.45 & 1.79 & 0.0017 & 0.025 \\
$\mathrm{~S} / \mathrm{Si} \ldots$. & $1.0 \pm 0.4$ & 1.28 & 0.96 & 0.24 & 1.5 & 1.4 \\
$\mathrm{Fe} / \mathrm{Si} \ldots$ & $<0.018$ & 0.88 & 0.13 & 0.15 & 0.89 & 0.91 \\
\hline
\end{tabular}

${ }^{a}$ Woosley \& Weaver (1995). ${ }^{b}$ Pulsating delayed detonation (PDDe) and delayed detonation (DDTe) models from Badenes et al. (2003).

(namely IC 443 and W49B) out of a sample of 6, some ions are indeed overionized. Such an ionization state of the plasma has many important implications, since it would indicate rapidly cooling plasma due, for instance, to thermal conduction. A thermally conductive model has been invoked to explain the centrally peaked morphology of MM SNRs (Cox et al. 1999; Shelton et al. 1999), and overionization would provide a nice consistency check.

We investigated whether overionization conditions are consistent with our XMM-Newton data, performing our check in the same regions of IC 443 analyzed by Kawasaki et al. (2002). In the "North" region, we obtained that overionization is not consistent with the results for the $\mathrm{S}$ ions. In the "Center" region, overionization is still possible though not required at the $90 \%$ confidence level, for the same ions. We point out that this region is characterized by a wide spread of $E W \mathrm{~s}$ in both the $\mathrm{Si}$ and the $\mathrm{S}$ maps $(\triangle E W /\langle E W\rangle \sim 40 \%)$, therefore the analyzed spectrum originates from chemically inhomogeneous regions. A check of the ionization state would require more uniform conditions. For instance, Miceli et al. (2006) found that in the central core of the SNR W49B there is no compelling evidence of overionization. Unfortunately, our attempt to analyze smaller but more homogeneous regions was affected by the low statistics, and did not significatively constrain the spectral parameters.

\subsection{Constraints on the SN progenitor}

We presented in Sects. 3.2, 3.3 high resolution maps of some properties of the hot plasma (median photon energy, and $E W \mathrm{~s}$ of $\mathrm{Si}$ and $\mathrm{S}$ lines). We further investigated and quantified the properties of the X-ray emitting plasma through a spatially-resolved spectral analysis (Sect. 3.4). These results allowed us to infer the spatial distribution of the stellar ejecta, and to estimate their abundance pattern and masses in the ring structure, which are strong clues to the nature of the SN progenitor (e.g. Rakowski et al. 2006; Miceli et al. 2006; Park et al. 2004), and the age of the remnant (see Sect. 4.3).

Table 3 reports the best fit metal abundances of the "ring" region relative to $\mathrm{Si}$ (Col. 1), the abundance ratios expected for core-collapse SNe with different progenitor masses (Cols. 2-4; Woosley \& Weaver 1995), and for type Ia SNe according to different deflagration models (Cols. 5, 6; Rakowski et al. 2006; Badenes et al. 2003). Bearing in mind the uncertainty in the predictions of nucleosynthesis yields, our comparison is aimed at discriminating between type Ia and type II SN explosions. Any further inference, for example on the mass of the progenitor, would be highly dependent on the adopted model.

The very low Fe abundance cannot meaningfully constrain the progenitor explosion, and it can be explained if the innermost ejecta layers have not been shocked yet. The high $\mathrm{Mg}$ abundance allowed us to disfavor a type Ia SN, which primarily produces Fe-group elements and a negligible fraction of lighter metals. A type II SN was previously suggested as the remnant progenitor, 
only on the basis of its location in a star-forming region and the putative association with a PWN (Gaensler et al. 2006; Bocchino \& Bykov 2001; Olbert et al. 2001). Our results further support and strengthen a core-collapse origin for IC 443.

In order to estimate the total mass of the ejecta, we assumed that the ejecta are confined within a shell of radius $R_{\mathrm{ej}} \sim 4.5 \mathrm{pc}$, and width $\Delta r \sim 1 \mathrm{pc}$, for a total emission volume $V_{\text {shell }} \sim 7.7 \times$ $10^{57} \mathrm{~cm}^{3}$. Hence the hot plasma fills the entire volume $V_{\text {shell }}$ (filling factor $f_{\mathrm{h}}=1$ ). The above assumption might not be valid for the $\mathrm{Mg}$ ejecta, since it is based on the $E W$ maps of Si and S line complexes. Such high- $Z$ elements are thought to be synthesized by explosive O- or Si-burning in the core of a massive star, while lighter metals mostly reside in the outer layers. If the stellar stratification persists after the explosion, core-collapse nucleosynthesis models predict the $\mathrm{Mg}$ ejecta to be preferentially located outside the Si shell (cf. Fig. 1 of Thielemann et al. 1996), thus occupying a larger volume. The possibility of mixing or overturning of the ejecta layers (Willingale et al. 2002; Hwang et al. 2000) adds further uncertainty. Therefore the estimated mass of $\mathrm{Mg}$ should be taken with care.

For the case where the electrons are primarily from $\mathrm{H} / \mathrm{He}$ $\left(n_{\mathrm{e}}=1.2 n_{\mathrm{H}}\right)$, we derived a hot component gas density of $n_{\mathrm{H}}=$ $(0.62 \pm 0.10) \mathrm{cm}^{-3}$ in our "ring" region, which intercepts a line of sight of $\sim 2$ pc. By assuming the same line of sight for the inner and outer regions, we estimated a density of $n_{\mathrm{H}}=(0.60 \pm$ $0.10) \mathrm{cm}^{-3}$ and $n_{\mathrm{H}}=(0.96 \pm 0.16) \mathrm{cm}^{-3}$, respectively.

We extrapolated the density derived in the "ring" region to the whole shell of ejecta, thus estimating the total mass of hot metal-rich plasma, $M_{\text {tot }} \sim 4 M_{\odot}$. We calculated a lower limit on the mass of ejecta $M_{Z}$ as $M_{Z} / M_{\text {tot }} \geq A_{Z} \times\left(M_{Z} / M_{\text {tot }}\right)_{\odot}$ (e.g. Lazendic et al. 2005), where $M_{\text {tot }}$ is the total mass of the hot X-ray-emitting plasma, $A_{Z}$ is the measured abundance for the element $Z$, and $\left(M_{Z} / M_{\text {tot }}\right)_{\odot}$ is the standard solar mass fraction $\left(6.4 \times 10^{-4}\right.$ for $\mathrm{Mg}, 7.0 \times 10^{-4}$ for $\mathrm{Si}$, and $3.6 \times 10^{-4}$ for $\mathrm{S}$; Anders \& Grevesse 1989). We obtained: $M_{\mathrm{Mg}} \gtrsim 0.013 M_{\odot}, M_{\mathrm{Si}} \gtrsim$ $0.007 M_{\odot}, M_{\mathrm{S}} \gtrsim 0.003 M_{\odot}$.

An upper limit on $M_{Z}$ was derived by the assumption of "pure" Z-ejecta, i.e. stellar fragments in which all the electrons are provided by the ionized specie $Z, n_{\mathrm{e}} \sim K_{Z} n_{Z}$. This is a crude approximation of the ejecta mass, but not unrealistic as this kind of objects has already been observed in other SNRs, such as the Si-rich knots in Cas A (Laming \& Hwang 2003). We considered the contribution from both the ionization states of $\mathrm{Si}$ and $\mathrm{S}$ $\left(K_{\mathrm{Si}}=12.5, K_{\mathrm{S}}=14.5\right)$, but only the highest ionization state of $\mathrm{Mg}\left(K_{\mathrm{Mg}}=11\right)$, as results from the best fit model (see Fig. 5, middle panel). We obtained: $M_{\mathrm{Mg}} \lesssim 0.55 M_{\odot}, M_{\mathrm{Si}} \lesssim 0.30 M_{\odot}$, $M_{\mathrm{S}} \lesssim 0.15 M_{\odot}$.

According to Woosley \& Weaver (1995) the predicted masses of $\mathrm{Mg}, \mathrm{Si}$ and $\mathrm{S}$ ejecta lie between $0.01-0.4 M_{\odot}$, $0.02-0.4 M_{\odot}$, and $0.01-0.2 M_{\odot}$, respectively. These values agree well with the ranges derived from our analysis, which can be considered meaningful constraints on the ejecta masses in the hot shell.

\subsection{Age of IC 443}

We examined the case of a SNR expanding in an uniform ambient medium $(s=0)$ and with an uniform ejecta distribution $(n=0)$. Similar results are found when considering a power law ejecta distribution $(n=2)$. We took our estimate of the pre-shock density in the eastern regions (subshell B), $n_{0} \sim 0.25 \mathrm{~cm}^{-3}$, and the mass of the hot plasma calculated in Sect. 4.2. For typical $\mathrm{SN}$ energies $\left(\sim 10^{51} \mathrm{erg}\right)$, the length scale and the time scale of

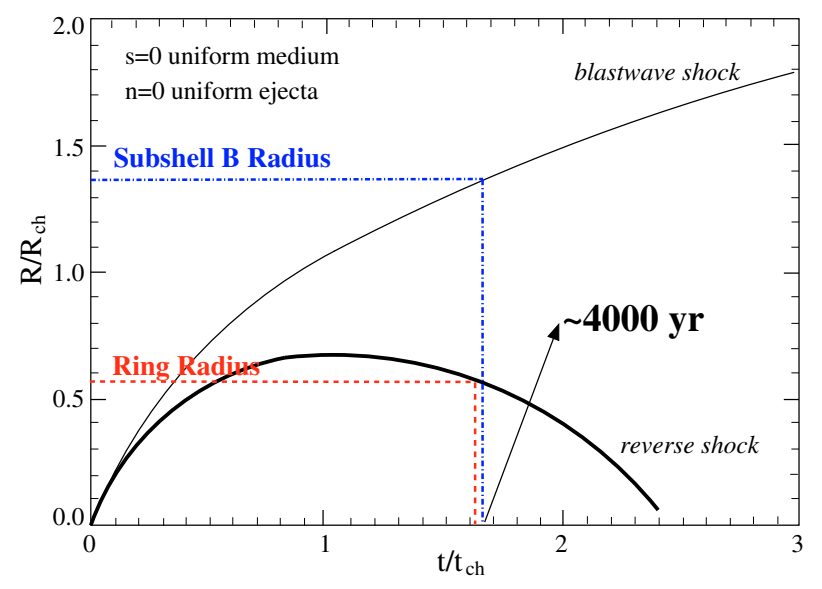

Fig. 7. Shock positions for blast-wave (thin solid line) and reverse (thick solid line) shocks in remnants of uniform ejecta and evolving in an uniform medium (Truelove \& McKee 1999). A comparison with the observed values for IC $443\left(r_{1} \sim 11 \mathrm{pc}\right.$ for the subshell $\mathrm{B}$, and $r_{2} \sim 4.5 \mathrm{pc}$ for the ring of hot gas, tracing the forward and reverse shock location, respectively) gives a SNR age of $4 \times 10^{3} \mathrm{yr}$.

the Truelove \& McKee (1999) solution are $R_{\mathrm{ch}} \sim 8 \mathrm{pc}$ and $t_{\mathrm{ch}} \sim$ $2.3 \times 10^{3} \mathrm{yrs}$, respectively.

Figure 7 shows the predictions of Truelove \& McKee (1999) compared with the observed parameters of IC 443: a forward shock radius of $11 \mathrm{pc}$ (shell $\mathrm{B}$ radius) and a reverse shock radius of $4.5 \mathrm{pc}$ (ring radius), correspond to an evolutive age of $\sim 4000 \mathrm{yr}$, much younger than some previous estimates. In particular, Chevalier (1999) derived an age of $3 \times 10^{4} \mathrm{yr}$ by assuming that the remnant has been evolving in a very dense environment $\left(\sim 15 \mathrm{~cm}^{-3}\right)$ since its early stages. Our result is instead more similar to the age estimated by Petre et al. (1988), who considered an exponential density distribution for the circumstellar environment. The analysis of the soft X-ray emission from IC 443, and the derived density profile (cf. Troja et al. 2006, Fig. 7) suggest that the blast wave propagated in a much lower density medium, and only recently has encountered a sharp density gradient in the NE (subshell A), impacting onto a neutral hydrogen cloud (Rho et al. 2001; Denoyer 1978).

As derived in Sect. 4.2, the remnant likely originated from the collapse of a massive star. Hence, during the progenitor lifetime strong stellar winds could have blown away the surrounding molecular clouds, forming a low density cavity, where the remnant is now evolving. Braun \& Strom (1986) first argued that the multiple shell morphology of IC 443 might be the result of such pre-SN interaction, and that the blast wave is impacting on the pre-existing wind-bubble walls. A detailed kinematical study of the optical filaments in IC 443, carried out by Meaburn et al. (1990), gave further support to the scenario proposed by Braun \& Strom (1986). The authors detected high velocity features along their slit Pos. 9, at the edge between subshell A and B, which can be easily explained if the emitting material, accelerated by the passage of the shock, is seen edge-on. The resultant geometry of the optical filaments is fully consistent with an SNR evolving within pre-existing interconnected cavities (cf. Meaburn et al. 1990, Fig. 5).

Since the medium surrounding massive stars is considerably modified by pre-SN winds (e.g. Chu et al. 2003; Dwarkadas 2005), we verified whether our estimate of the age of IC 443 significantly depends on the assumption of a uniform ambient medium. For this purpose, we modeled the evolution of a SNR in an isothermal medium with a power-law density profile $\rho \propto r^{-2}$, 
by performing a numerical hydrodynamic simulation. We used the FLASH code (Fryxell 2000) and we set up a spherically symmetric explosion of $3 M_{\odot}$ of ejecta with a total energy of $10^{51} \mathrm{erg}$. The initial velocity profile of the ejecta increases linearly with their distance from the center, and their density profile decreases exponentially (in agreement with Wang \& Chevalier 2002). In this case $\rho_{\mathrm{s}}=\dot{M}_{\mathrm{w}} /\left(4 \pi v_{\mathrm{w}}\right) \sim 1.76 \times 10^{14} \mathrm{~g} \mathrm{~cm}^{-1}$, where $\dot{M}_{\mathrm{w}}$ is the mass-loss rate of the wind and $v_{\mathrm{w}}$ is its speed, and both are assumed to be constant. The location of the forward and reverse shock fronts obtained in the simulation suggests a slightly younger age $(\sim 3000 \mathrm{yr})$. Our basic assumption, i.e. that the radial separation between the forward and the reverse shock is the result of the SNR evolutive stage, does not hold if the forward shock in the NW has been strongly decelerated in the cavity walls. Modifications of the shock velocity, due to the interaction of the blast wave with the confining walls, led to systematically underestimate the SNR age. In that case, the inferred value of $4000 \mathrm{yr}$ only provides a lower limit. However, in such a scenario, a close correlation between the two radii, as shown in Fig. 7, is not expected, as the forward shock radius would mainly depend on the properties of the cavity wall. We therefore conclude that the morphology of the X-ray emitting plasma in IC 443 robustly suggests an age $<10^{4} \mathrm{yr}$.

\subsubsection{Implications for the PWN}

A detailed study of the morphological and spectral properties of the PWN, and its central neutron star (NS; J0617+2221) has been presented by Gaensler et al. (2006). They inferred a blackbody temperature $k T^{\infty} \sim 100 \mathrm{eV}$ for the NS, and argued that the properties of the thermal radiation from the NS are consistent with the Chevalier (1999) age of 30000 yrs. By using the theoretical cooling curve of Kaminker et al. (2002), we checked that our new estimate of the SNR age and the temperature range measured by Gaensler et al. (2006) are still consistent with the cooling of a $1.35-1.45 M_{\odot}$ NS with a 1 p proton superfluid core. Thus, the present knowledge of NS cooling curves does not allow us to place tighter constraints on the NS age that would strengthen the association between the remnant and the PWN. The NS temperature of Gaensler et al. (2006) and a putative age $<10^{4} \mathrm{yr}$ are in agreement with observational results from other young NSs: PSR J1119-6127 ( 1.6 kyr) RX J0822-43 ( 3.7 kyr) 1E 1207-52, and PSR J1357-6429 ( 7 kyr; Zavlin 2007b,a; Kaminker et al. 2002).

The location of the ring of hot metal-rich plasma in our Figs. 2 and 4, just around the PWN, is an additional argument in support of the physical connection between the PWN and IC 443. In the plane of the image (see e.g. Fig. 2) only the south side of the PWN seems to be in contact with the reverse shock, suggesting that the PWN may be in the reverse shock or subsonic expansion stage, but not in the high supersonic stage (van der Swaluw et al. 2004). This is consistent with an age of few thousand years, and is in rough agreement with the mildly supersonic Mach value found by Gaensler et al. (2006).

The orientation of the cometary tail and the position of the PWN cannot be straightforwardly explained. This mismatch is still an open issue, but it does not precludes the association between the PWN, the ring and the SNR (see also Gaensler et al. 2006). For instance, a similar apparent offset between the stellar remnant, the forward and the reverse shock geometric centers has been already observed in Cas A (Gotthelf et al. 2001). A possibility is that strong reverse shocks, generated by the interaction with the surrounding clouds, skewed the orientation of the nebula. An asymmetric SN explosion or the presence of strong density gradients across the remnant offer a valid explanation for the observed offsets (Gotthelf et al. 2001; Reed et al. 1995). Another possible explanation is that the SN ejecta have been decelerated by the southern ridge of molecular material, and, as a consequence, the geometric center of the ring appears shifted toward the north direction.

\section{Conclusions}

We analyzed the hard (1.4-5.0 keV) X-ray emission of the supernova remnant IC 443 , as observed by a set of public archive $X M M-N e w t o n$ observations. Unlike the soft X-ray emission, which directly traces the interaction with the surrounding clouds (Troja et al. 2006), the hard X-ray emission has a centrallypeaked morphology, reminiscent of the subclass of mixed morphology SNRs. The presence of a ring-shaped feature, which encircles the PWN, has been revealed in the equivalent width maps of Si and S lines (Fig. 3).

A spatially-resolved spectral analysis confirms that this structure is associated with hot metal rich plasma, whose abundances are consistent with a core-collapse SN origin, but not with an exploding white dwarf. We argue that the ring indicates the location of an ejecta layer heated by the reverse shock, and that the reverse shock has partially hit the PWN. Dynamically, the location of the ejecta ring suggests an age of $\sim 4000 \mathrm{yr}$ for IC 443, an order of magnitude lower than some previous estimates, based on expansion in a very dense environment. A younger age is indeed more consistent with an SNR expansion within wind-blown bubble shells surrounded by dense clouds, as first suggested by Braun \& Strom (1986) and recently considered by Troja et al. (2006).

We detect only marginal evidence of plasma overionization in the NE part of the remnant. The bright hard 1.4-5.0 keV $\mathrm{X}$-ray emission in the NE region of IC 443 also shows high temperatures and high metal abundances, as shown in Troja et al. (2006). More detailed results from this region will be reported in a future work.

Acknowledgements. We thank the anonymous referee for his/her insightful comments on this paper. We also thank J. Raymond and R. Chevalier for useful discussions about the optical and the X-ray emission of IC 443, and Richard Owen for his kind help. F.B. and M.M. acknowledge financial contribution from the contract ASI-INAF I/203/05. The software used in this work was in part developed by the DOE-supported ASC / Alliance Center for Astrophysical Thermonuclear Flashes at the University of Chicago. This work makes use of results produced by the PI2S2 Project managed by the Consorzio COMETA, a project co-funded by the Italian Ministry of University and Research (MIUR) within the Piano Operativo Nazionale "Ricerca Scientifica, Sviluppo Tecnologico, Alta Formazione" (PON 2000-2006). More information is available at http://www.pi2s2.it and http://www. consorzio-cometa.it.

\section{References}

Albert, J., Aliu, E., Anderhub, H., et al. 2007, ApJ, 664, L87

Anders, E., \& Grevesse, N. 1989, Geochim. Cosmochim. Acta, 53, 197

Arnaud, K. A. 1996, in Astronomical Data Analysis Software and Systems V, ASP Conf. Ser., 101, 17

Asaoka, I., \& Aschenbach, B. 1994, A\&A, 284, 573

Badenes, C., Bravo, E., Borkowski, K. J., \& Domi'nguez, I. 2003, ApJ, 593, 358 Bocchino, F., \& Bykov, A. M. 2000, A\&A, 362, L29

Bocchino, F., \& Bykov, A. M. 2001, A\&A, 376, 248

Bocchino, F., \& Bykov, A. M. 2003, A\&A, 400, 203

Braun, R., \& Strom, R. G. 1986, A\&A, 164, 193

Burton, M. G., Hollenbach, D. J., Haas, M. R., \& Erickson, E. F. 1990, ApJ, 355, 197

Bykov, A. M., et al. 2008, ApJ, in press [arXiv : 0801.1255]

Bykov, A. M., Bocchino, F., \& Pavlov, G. G. 2005, ApJ, 624, L41

Cassam-Chenaï, G., Decourchelle, A., Ballet, J., et al. 2004, A\&A, 414, 545 
Chevalier, R. A. 1999, ApJ, 511, 798

Chu, Y.-H., Gruendl, R. A., \& Guerrero, M. A. 2003, in Rev. Mex. Astron. Astrofis. 27, ed. J. Arthur, \& W. J. Henney, Rev. Mex. Astron. Astrofis. Conf. Ser., 15,62

Claussen, M. J., Frail, D. A., Goss, W. M., \& Gaume, R. A. 1997, ApJ, 489, 143

Cornett, R. H., Chin, G., \& Knapp, G. R. 1977, A\&A, 54, 889

Cox, D. P., Shelton, R. L., Maciejewski, W., et al. 1999, ApJ, 524, 179

Denoyer, L. K. 1977, ApJ, 212, 416

Denoyer, L. K. 1978, MNRAS, 183, 187

Dickel, J. R., Williamson, C. E., Mufson, S. L., \& Wood, C. A. 1989, AJ, 98, 1363

Duin, R. M., \& van der Laan, H. 1975, A\&A, 40, 111

Dwarkadas, V. V. 2005, ApJ, 630, 892

Esposito, J. A., Sreekumar, P., Hunter, S. D., \& Kanbach, G. 1996, BAAS, 28, 1346

Gaensler, B. M., Chatterjee, S., Slane, P. O., et al. 2006, ApJ, 648, 1037

Gotthelf, E. V., Koralesky, B., Rudnick, L., et al. 2001, ApJ, 552, L39

Hewitt, J. W., Yusef-Zadeh, F., Wardle, M., Roberts, D. A., \& Kassim, N. E. 2006, ApJ, 652, 1288

Hoffman, I. M., Goss, W. M., Brogan, C. L., Claussen, M. J., \& Richards, A. M. S. 2003, ApJ, 583, 272

Hughes, J. P., Ghavamian, P., Rakowski, C. E., \& Slane, P. O. 2003, ApJ, 582, L95

Hwang, U., Holt, S. S., \& Petre, R. 2000, ApJ, 537, L119

Kaminker, A. D., Yakovlev, D. G., \& Gnedin, O. Y. 2002, A\&A, 383, 1076

Kawasaki, M., Ozaki, M., Nagase, F., Inoue, H., \& Petre, R. 2005, ApJ, 631, 935

Kawasaki, M. T., Ozaki, M., Nagase, F., et al. 2002, ApJ, 572, 897

Laming, J. M., \& Hwang, U. 2003, ApJ, 597, 347

Lampton, M., Margon, B., \& Bowyer, S. 1976, ApJ, 208, 177

Lazendic, J. S., \& Slane, P. O. 2006, ApJ, 647, 350

Lazendic, J. S., Slane, P. O., Hughes, J. P., Chen, Y., \& Dame, T. M. 2005, ApJ, 618,733

Leahy, D. A. 2004, AJ, 127, 2277

Mavromatakis, F., Aschenbach, B., Boumis, P., \& Papamastorakis, J. 2004, A\&A, 415, 1051
Meaburn, J., Whitehead, M. J., Raymond, J. C., Clayton, C. A., \& Marston, A. P. 1990, A\&A, 227, 191

Miceli, M., Decourchelle, A., Ballet, J., et al. 2006, A\&A, 453, 567

Neufeld, D., \& Yuan, Y. 2008, ApJ, in press [arXiv: 0801.2141]

Olbert, C. M., Clearfield, C. R., Williams, N. E., Keohane, J. W., \& Frail, D. A. 2001, ApJ, 554, L205

Park, S., Burrows, D. N., Garmire, G. P., et al. 2003a, ApJ, 586, 210

Park, S., Hughes, J. P., Slane, P. O., et al. 2003b, ApJ, 592, L41

Park, S., Hughes, J. P., Slane, P. O., et al. 2004, ApJ, 602, L33

Petre, R., Szymkowiak, A. E., Seward, F. D., \& Willingale, R. 1988, ApJ, 335, 215

Rakowski, C. E., Badenes, C., Gaensler, B. M., et al. 2006, ApJ, 646, 982

Reed, J. E., Hester, J. J., Fabian, A. C., \& Winkler, P. F. 1995, ApJ, 440, 706

Reich, W., Zhang, X., \& Fürst, E. 2003, A\&A, 408, 961

Rho, J., \& Petre, R. 1998, ApJ, 503, L167

Rho, J., Jarrett, T. H., Cutri, R. M., \& Reach, W. T. 2001, ApJ, 547, 885

Shelton, R. L., Cox, D. P., Maciejewski, W., et al. 1999, ApJ, 524, 192

Shelton, R. L., Kuntz, K. D., \& Petre, R. 2004, ApJ, 611, 906

Smith, R. K., Brickhouse, N. S., Liedahl, D. A., \& Raymond, J. C. 2001, ApJ, 556, L91

Snell, R. L., Hollenbach, D., Howe, J. E., et al. 2005, ApJ, 620, 758

Sturner, S. J., \& Dermer, C. D. 1995, A\&A, 293, L17

Thielemann, F.-K., Nomoto, K., \& Hashimoto, M.-A. 1996, ApJ, 460, 408

Troja, E., Bocchino, F., \& Reale, F. 2006, ApJ, 649, 258

Truelove, J. K., \& McKee, C. F. 1999, ApJS, 120, 299

van der Swaluw, E., Downes, T. P., \& Keegan, R. 2004, A\&A, 420, 937

van Dishoeck, E. F., Jansen, D. J., \& Phillips, T. G. 1993, A\&A, 279, 541

Wang, C.-Y., \& Chevalier, R. A. 2002, ApJ, 574, 155

Wang, Z. R., Asaoka, I., Hayakawa, S., \& Koyama, K. 1992, PASJ, 44, 303

Willingale, R., Bleeker, J. A. M., van der Heyden, K. J., Kaastra, J. S., \& Vink, J. 2002, A\&A, 381, 1039

Woosley, S. E., \& Weaver, T. A. 1995, ApJS, 101, 181

Zavlin, V. E. 2007a, ApJ, 665, L143

Zavlin, V. E. 2007b, Ap\&SS, 308, 297 